\title{
Human Errors Study Based on the Professionalization of Social Division Erke Tong ${ }^{a}$, Ripin Feng, and Jinwang Tang \\ (Air Force Airborne Academy, Guilin 541003, China) \\ aoakey@sina.com
}

\begin{abstract}
The professionalization of social division causes individual repetitive professional behavior, which is not neglect when we analyze reliable factors of human errors. Based on "S-O-R" model of behavioral psychology, this article analyzes the effects that professional behavior has on individual pattern of behaviors. Reliable factors of human errors are classified and analyzed, and a new analytical model of man-made error is put forward according to human professional behavior course.
\end{abstract}

Keywords: Human errors; Professionalization; Habitual errors; Individual errors.

\section{职业角度下的人为差错研究}

全二克，丰日品，唐金旺

( 空军空降兵学院, 广西桂林 541003 )

摘要: 社会分工职业化造成了个体重复性的职业行为, 在研究人为差错的形成原因时, 这种重复性的职业化行为不可忽 略。基于行为心理学原理, 分析了 “S-O-R” 模型中职业化行为对个体基本行为模式造成的影响, 将人为差错进行了归类分 析, 建立了职业角度下人为差错分析的基本模型。

关键词: 人为差错; 职业化; 习惯差错; 个体差错

中图分类号: X928.03 文摘标识码: A

\section{1 引言}

所谓人为差错, 指的是非主观目的造成行为结果偏离目标标准的行为, 人为差错具有非主观目的性和 不确定性的特点。自从 Sandia 实验室进行的研究揭开了人为差错研究的第一页, 中外学者对人为差错进 行了诸多深入的研究, 比较典型的有人因失误率预测法（THERP）、人的认知可靠性模型（HCR）、成功可 能性权重法 (SLIM) 、人行为可靠系统评测法 (SHARP) 等。总体归纳, 这些研究具有三个基本特征:

（1）将人群定义在差错事件的片段之中。将研究定格在样本人群在具体事件中的行为表现、行为致 因和行为流程, 对样本人群在事件中的可靠性和行为影响因素进行分析, 如建立失误数据库、人的认知可 靠性模型（HCR）、DNE 直接数据统计法、OAT 方法。

(2) 研究对象为任务事件本身。研究某一事件出现人为差错的统计概率，对任务事件的执行环节、 影响因素进行分析, 从任务的认知、执行流程、环境影响等方面寻求解决方案, 如人因失误率预测法 （THERP）、ASEP 事故后果评估法、成功可能性权重法（SLIM）就是利用人行为形成因子 (PSFs) 对人的 失误概率进行预测。

(3) 研究对象为一般人群。即研究普遍的人群, 而不考虑特定的个体。 


\section{2 人为差错职业角度分析的基本思想}

人为差错, 指的是非主观目的造成行为结果偏离目标标准的行为, 人为差错具有非主观目的性和不确 定性的特点。自从 Sandia 实验室进行的研究揭开了人为差错研究的第一页, 当前人为差错研究比较典型 的有: 人因失误率预测法 (THERP) 、人的认知可靠性模型 (HCR) 、成功可能性权重法 (SLIM)、人行为 可靠系统评测法 (SHARP) 等。总体归纳, 这些研究具有三个基本特征:

(1) 将人群定义在差错事件的片段之中。将研究定格在样本人群在具体事件中的行为表现、行为致 因和行为流程, 对样本人群在事件中的可靠性和行为影响因素进行分析, 如建立失误数据库、人的认知可 靠性模型（HCR）、DNE 直接数据统计法、OAT 方法。

(2) 研究对象为任务事件本身。研究某一事件出现人为差错的统计概率, 对任务事件的执行环节、 影响因素进行分析, 从任务的认知、执行流程、环境影响等方面寻求解决方案, 如人因失误率预测法 （THERP）、ASEP 事故后果评估法、成功可能性权重法（SLIM）就是利用人行为形成因子 (PSFs) 对人的 失误概率进行预测。

(3) 研究人群为一般的人群, 而不考虑特定的个体。

\section{3 职业角度下人为差错的类型分析}

\section{1 职业分析的基本框架}

在社会任何职业中，一次生产或社会活动必须要经过技能贮备、任务执行、结果输出的过程。

（1）技能它备。在职业化条件下，行为者首先应当具备基本的任务技能方可执行任务，比如工人要 经过培训才能上岗, 教师、医生、护士都需要具备执业资格证等等, 这是一个基本的职业原则。技能咜备 包括两个方面:

操作技能 (Technique) : 熟悉任务的执行流程, 具备合格的操作能力, 能够对一般的任务情况进行 正确处置;

认知能力 (Cognition) : 对任务有一定的知识, 掌握任务的基本规律, 能够判断任务的性质并选择 正确的应对措施。

(2) 任务执行。行为心理学家提出了行为的基本模式 “刺激 (Stimulus) -思维 (Organism)-行动 （Response）” 即 “S- $0-\mathrm{R}$ ” 模式, 这是执行任务的基本过程。

(3) 结果输出。系统对任务执行的结果反馈和评价。

由此建立职业任务过程基本模型如下:

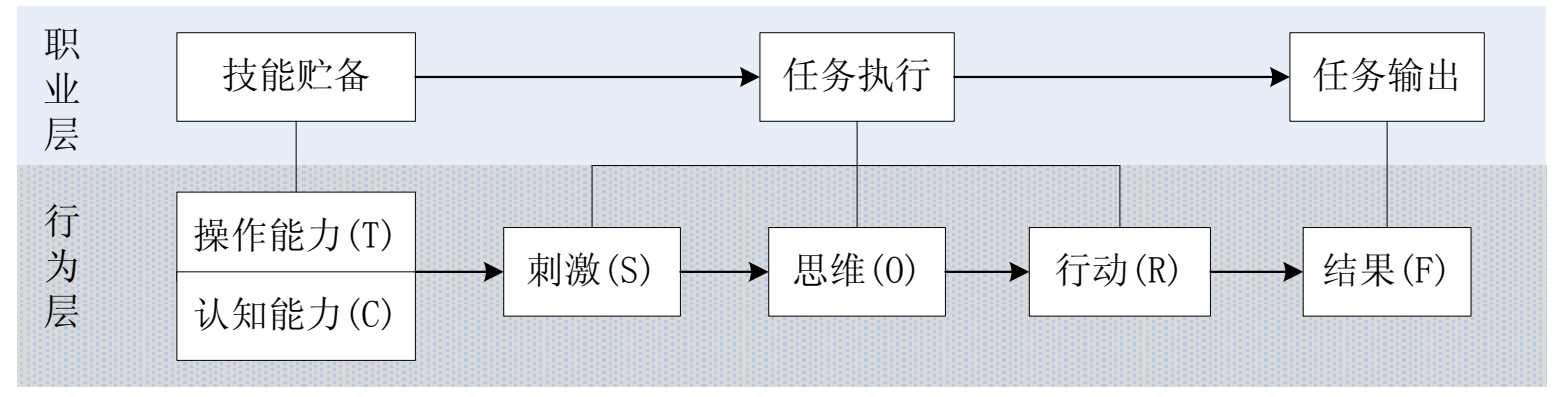

图 1. 职业化过程基本模型 


\section{2 人为差错的基本分类}

（1）操作差错。由于操作水平达不到标准而导致的差错, 如技术不熟练、动作流程错误等等。

（2）认知差错。由于对任务情景的认识未达到标准, 导致对任务的诊断发生偏差而造成的差错, 如 分析判断差错、对关键环节理解不正确、决策失误等。

(3) 习惯差错。当任务的执行过程多次重复形成思维定式或习惯性操作，而任务的输入发生改变后 仍然按习惯进行操作, 从而导致行为差错。形成习惯差错有三个要素: “ $S-0-R$ ” 模式多次重复, 形成 “ $S-R$ ” 自动化阶段, 意识控制不再起主导性作用; 长期出现的重复刺激使行为者的注意力下降; 一旦 $\mathrm{S}$ 发生变化, 依然按照 “S-R” 操作处置, 将形成执行差错。

（4）个体差错。由于个体对任务环境的适应能力、应激能力达不到标准或生活情景影响, 而导致的 诊断或操作偏差。每个人对信息的接受能力、对环境的应激能力、对压力的适应能力是不同的, 有个人的 极限, 个体在不合适的岗位上发生差错的概率将会增大; 个体的生活状况和生理节奏波动（如情感、家庭、 身体因素）会对任务的思维和操作造成影响。

（5）系统差错。系统差错是任务背景的设计，不属于人为差错的内容，但重复出现的系统差错将会 对人的认知和行为产生影响。

（6）随机差错。因不可控因素出现的不确定差错。

\section{3 人为差错的职业分析方法}

设个体的操作能力合格和不合格分别记为 $T$ 和 $\overline{\mathbf{T}}$, 认知能力合格和不合格分别记为 $\mathrm{C}$ 和 $\overline{\mathbf{C}}$ 。任务事件 为 $S$, 正确思维和错误思维分别为 0 和 $\overline{\mathbf{0}}$, 正确行动和错误行动分别为 $R$ 和 $\overline{\mathbf{R}}$, 正确结果和错误结果分别 为 $\mathrm{Y}$ 和 $\mathrm{N}$ 。

（1）任务执行的正常状态是: $T \cap C \rightarrow S \rightarrow 0 \rightarrow R \rightarrow Y$ 。即操作能力和认知能力均达到标准的前提下, 刺 激 $\mathrm{A}$ 出现, 通过正确的思维判断, 并执行了正确的行动, 结果输出正确。

（2）操作差错: $\overline{\mathbf{T}} \cap \mathrm{C} \rightarrow \mathrm{S} \rightarrow 0 \rightarrow \overline{\mathbf{R}} \rightarrow \mathrm{N}$ 。在操作技能非达标情况下, 刺激 $\mathrm{S}$ 出现, 思维判断虽然正确, 但操作错误而导致结果差错, 其差错原因为操作差错。

（3）认知差错: $\mathrm{T} \cap \overline{\mathbf{C}} \rightarrow \mathrm{S} \rightarrow \overline{\mathbf{0}} \rightarrow \overline{\mathbf{R}}_{\rightarrow} \mathrm{N}$ 。在操作能力达标情况下, 对任务的背景认知未达到标准, 刺 激 $\mathrm{S}$ 出现, 思维判断错误 $\overline{\mathbf{0}}$, 执行了错误的行动, 导致结果差错, 此差错原因为认知差错。

（4）习惯差错: $\mathrm{T} \cap \mathrm{C} \rightarrow \overline{\mathbf{S}}_{\boldsymbol{B}} \rightarrow \mathrm{R} \rightarrow \mathrm{N}$ 。操作能力和认知能力均达到标准情况下, 任务事件 $\mathrm{S}$ 发生了改变,

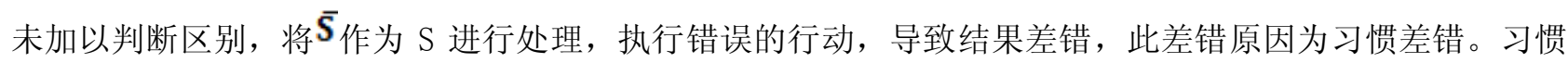
差错的基本特点是: 差错者并非技术或能力原因造成, 而是技术和能力较好的职业者; 外在表现为大意、 疏忽、注意力不集中、不重视、走过场; 差错无法正常预测。

（5）个体差错: $\mathrm{T} \cap \mathrm{C} \rightarrow \mathrm{S} \rightarrow 0(\overline{\mathbf{0}}) \rightarrow \overline{\mathbf{R}} \rightarrow \mathrm{N}$ 。在操作能力和认知能力均达到标准情况下, 刺激 $\mathrm{S}$ 出现, 判断正确而执行了错误的操作或判断错误而导致差错, 其原因为个体差错。个体差错通常是由于行为者情 绪、心理或身体状态波动形成的, 其特点是: 差错者为经过长期工作的职业者; 相对差错频率较大; 情绪、 状态反常。 
(6) 系统差错。正确的行动引起正确的结果, 错误的行动引起错误的结果, 如 $S \rightarrow R \rightarrow Y, S \rightarrow \overline{\mathbf{R}} \rightarrow N$ 。 若结果相反, 可能为系统差错; 若不正常的流程反复出现, 如 $S \rightarrow \overline{\mathbf{R}} \rightarrow Y$ 或 $S \rightarrow R \rightarrow N$, 则这种不正常流程可 能会得到强化并形成习惯差错，将会使认知模式和操作模式发生迁移。

\section{4 结语}

本文对职业化形成过程中的人为差错成因进行了探究, 并建立了分析模型。现代社会生产中, 教育和 培训的价值已被充分认识, 技能和知识的职业化普遍形成, 操作差错和认知差错最易被发现、识别和预防。 相反来说, 习惯差错和个体差错掩藏在操作差错和认知差错之下, 最终表现为某一类具体操作或认知的显 性形式, 诸如不熟练、疏忽或精力不集中。因此在安全工作中, 应结合根据个体职业特征深入分析差错类 型, 进一步探索有效的预防对策。

\section{参考文献}

[1］Hollage1 E. 认知可靠性和误差分析方法 [M]. 爱思唯尔科学有限公司, 1998.

[2]沈彩华. 空中交通管制中人为错误的影响和预防措施[J]. 科技创新导报, 2014（15）：235-236.

[3] 李涛, 蒋英业, 孙志英等. 基于灰色关联分析法分析航空维修误差模式与其停顿之间的关系 [J]. 中国安全科学 2011, 21 (2) :85-89.

[4]高东岳, 王一寿, Gorgin RAHIM. 全尺寸复合水平尾部的在役结构健康监测 $[J]$. 武汉理工大学学报 (材料科学版), 2015 (06) : 1215-1224.

[5]杨长奇, 何伟. 基于概率安全评价方法评估空中交通管制操作中的人体安全 $[J]$. 运输研究进展, 2015：46-51。

\section{References}

[1] Hollagel E, Cognitive Reliability and Error Analysis Method [M], Elseiver Science Ltd, 1998.

[2] Shen Caihna. The influence and prevention of human error in air traffic control [J]. Science and Technology Innovation Herald, 2014(15).

[3] LI Tao, JIANG Yingyie, SUN Zhiying, et al. Analysis of relationships between aviation maintenance error modes and their pauses based on grey relational analysis method. China Safety Science Journal, 2011, 21(2):85-89.

[4] GAO Dongyue, WANG Yishou, Gorgin RAHIM. In-service Structural Health Monitoring of a Full-scale Composite Horizontal Tail [J]. Journal of Wuhan University of Technology (Materials Science Edition), 2015(06).

[5] Yang Chang-qi, He Wei. Human safety assessment in air traffic control operation based on the method of probabilistic safety assessment, Advances in Transportation Studies, 2015, 46-51. 